\title{
Multiobjective combinatorial optimization for performance evaluation by a meta- heuristic satisficing tradeoff method
}

\author{
Hiroyuki Tamura \\ Department of Systems and Human Science \\ Graduate School of Engineering Science \\ Osaka University \\ 1-3 Machikaneyama, Toyonaka \\ Osaka 560-8531, JAPAN \\ TEL+81-6-6850-6375 FAX+81-6-6850-6341 \\ tamura@sys.es.osaka-u.ac.jp \\ Tomohiro Shibata \\ Murata Corporation \\ Yasu, Shiga, JAPAN \\ Itsuo Hatono \\ Information Processing Center \\ Kobe University \\ Kobe, JAPAN
}

\begin{abstract}
In this paper an effective meta-heuristic approach is proposed to realize a satisficing tradeoff method for solving multiobjective combinatorial optimization problems of performance evaluation. Firstly, Pareto optimal solutions (individuals) are generated by using a genetic algorithm with family elitist concept for a multiobjective combinatorial optimization problem. Then, we try to find a preferred solution of the decision maker based on the satisficing tradeoff method. Usually, a conventional satisficing tradeoff method needs to solve a complex min-max problem in each iteration of the algorithm for a given aspiration level of each objective function. The min-max problem is to minimize maximum value of a regularized regret function. In this paper a new meta-heuristic satisficing tradeoff method is proposed in which we do not need to solve a complex min-max problem in each iteration, but we try to find a min-max solution in the Pareto optimal solutions (individuals) generated by the genetic algorithm. We further revise the min-max solution by using a local search approach such as a simulated annealing method. As a numerical example a flowshop scheduling problem is included to verify the effectiveness of the method proposed in this paper.
\end{abstract}

Keywords

Performance evaluation, production management, multiobjective combinatorial optimization, meta-heuristic approach, satisficing tradeoff method, flowshop scheduling 


\section{INTRODUCTION}

In advanced production management systems performance evaluation is usually to be done under multiple objectives. Furthermore, in production scheduling, performance evaluation is to be done under combinatorial optimization. Since combinatorial optimization problems are usually NP-hard or sometimes NP-complete (Cook, et al., 1998), an optimal solution or even a suboptimal solution is hard to find even for a single-objective problem. Therefore, nobody has tried rigorously to solve multiobjective combinatorial optimization problems.

In this paper an effective meta-heuristic approach is proposed to realize a satisficing tradeoff method for solving multiobjective combinatorial optimization problems. Firstly, Pareto optimal solutions (individuals) are generated by using a genetic algorithm (Goldberg, 1989) with family elitist concept (Bedarahally, et al., 1996) for a multiobjective combinatorial optimization problem. Then, we try to find a preferred solution of the decision maker based on the satisficing tradeoff method. A conventional satisficing tradeoff method (Nakayama and Sawaragi, 1984) needs to solve a complex min-max problem in each iteration of the algorithm for a given aspiration level of each objective function. In this paper a new meta-heuristic satisficing tradeoff method is proposed in which we do not need to solve a complex min-max problem in each iteration, but we try to find a min-max solution in the Pareto optimal solutions (individuals) generated by the genetic algorithm. We further revise the min-max solution by using a local search approach such as a simulated annealing method. As a numerical example of a multiobjective combinatorial optimization problem a flowshop scheduling problem is included.

\section{SATISFICING TRADEOFF METHOD}

In general, performance evaluation problems in production management systems can be formulated as

minimize $f(x) \equiv\left(f_{1}(x), f_{2}(x), \ldots, f_{r}(x)\right)$

subject to $x \in X$

where $x$ denotes decision vector, $\mathrm{X}$ denotes the feasible region of the decision vector, $f_{i}(x), i=1,2, \ldots, r$ denotes multiple objective functions to be minimized, and $r$ denotes the number of objective funtions.

Basic algorithm of satisficing tradeoff method (Nakayama and Sawaragi, 1984) for solving multiobjective optimization problems can be written as follows:

(a) Specification of the range of each objective function:

Specify the ideal value $f_{i}^{*}$ and the nadir value $f_{i^{*}}$ for each objective function $f_{i}(x)$, $i=1,2, \ldots, r$ by minimizing and maximizing each objective function independently. 
(b) Specification of the aspiration level:

Ask the decision maker the aspiration level $f_{i}^{a}, i=1,2, \ldots, r$ for each objective.

(c) Solving min-max problem:

Let normalized weight for each objective be

$w_{i} \equiv \frac{1}{f_{i}^{*}-f_{i^{*}}}$

Solve min-max problem

$\operatorname{minimize}\left\{\max w_{i}\left(f_{i}(x)-f_{i}^{a}\right)\right\}$

$x \in X \quad 1 \leqq i \leqq r$

subject to $x \in X$

This mini-max problem is interpreted to minimize the maximum value of normalized dissatisfaction level as shown in Figure 1.

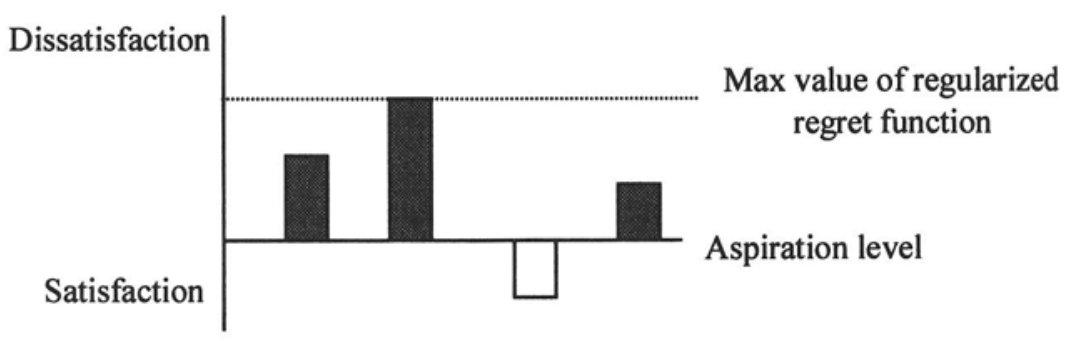

Figure 1 A min-max problem.

Instead of solving this min-max problem, an equivalent optimization problem

$\underset{x, z}{\operatorname{minimize}}\left\{z+\alpha \sum_{i=1}^{r} w_{i}\left(f_{i}(x)-f_{i}^{a}\right)\right\}$

subject to $\quad w_{i}\left(f_{i}(x)-f_{i}^{a}\right) \leqq z, \quad i=1,2, \ldots, r$

$$
x \in X
$$

is usually solved for small $\alpha$, say $10^{-6}$. Let $x$ be optimal solution to the minmax problem. 
(d) Tradeoff analysis:

Showing the solution $x$ and the resulting $f(x)$ to the decision maker we ask him if he would be satisfied with this solution. If he would not be satisfied with this solution, we ask him a new aspiration level for each objective function going back to (b).

\section{GENERATION OF A SET OF PARETO OPTIMAL SOLUTIONS (IN- DIVIDUALS) BY GENETIC ALGORITHM}

In this section we propose a method of generating a set of Pareto optimal solutions (nondominated solutions) of multiobjective optimization problems based on the family elitist strategy (Bedarahally, et al., 1996) in Genetic Algorithm (GA).

\subsection{Genetic Algorithm and Multiobjective Optimization}

GA (Holland, 1975; Goldberg, 1989) is one of the most promising evolutionary computation method in which the process of biological evolution is simulated. GA for a particular problem have the following five components:

(a) A genetic representation for a solution to the problem,

(b) A way to create an initial population of individuals which represent potential solutions,

(c) A function for evaluating fitness of the solutions,

(d) Genetic operators such as crossover, mutation and inversion that alter the composition of offsprings during the reproduction, and

(e) Parameter values that the GA uses, e.g. population size, number of generations, crossover rates, probability of mutation.

A significant advantage of GA for applying to multiobjective optimization problems is that GA can generate a set of Pareto optimal solution (individuals) simultaneously (Fonseca and Fleming, 1995; Tamaki and Nishino, 1998), where a Pareto optimal solution is a nondominated solution such that there exists no feasible solution which improves all the objective functions. Therefore, the decision maker has to tradeoff among multiple objective functions to improve some of them.

\subsection{Generation of a Set of Pareto Optimal Solutions (Individuals) by GA}

For applying GA to multiobjective optimization problems it is necessary to find an effective method for selecting Pareto optimal individuals in the current population. Several approaches have been proposed in this direction. Here, we propose a method to use family elitist strategy (Bedarahally, et al., 1996) in addition to parallel selection (Goldberg, 1989) and Pareto preservation strategy (Tamaki and Nishino, 1998).

(a) Parallel Selection:

Individuals of population are divided into sub-populations where the number of sub-populations is equal to $r$, the number of objective functions. Sub-popula- 
tions of the next generation are reproduced from the current population based on the value of each objective function.

(b) Pareto Preservation Strategy:

All the Pareto optimal individuals in a population at each generation are preserved in the next generation. If the number of Pareto optimal individuals would exceed the size of the population, parallel selection would be performed.

(c) Family Elitist Strategy:

Population is divided into several families. Each family contains $r$ individuals. Pairing, crossover, mutation and reproduction are performed in a family. Parallel selection and Pareto preservation strategy are used for reproduction.

Pareto preservation strategy for multiobjective optimization corresponds to elitism (Goldberg, 1989) for single-objective optimization, and non-dominated individuals contained in a population in each generation are all preserved in the next generation. By this strategy compromise solution could be obtained. Parallel selection from the Pareto optimal individuals enables us to improve each objective function further.

Family elitist strategy enables us to avoid the situation that the population is composed of strong individuals only. Therefore, we could expect to get Pareto optimal individuals from wider area of a set of feasible solutions without converging to unbalanced solution at the early stage. Figure 2 shows a family elitist concept used in GA and Figure 3 shows genetic operations in the families.

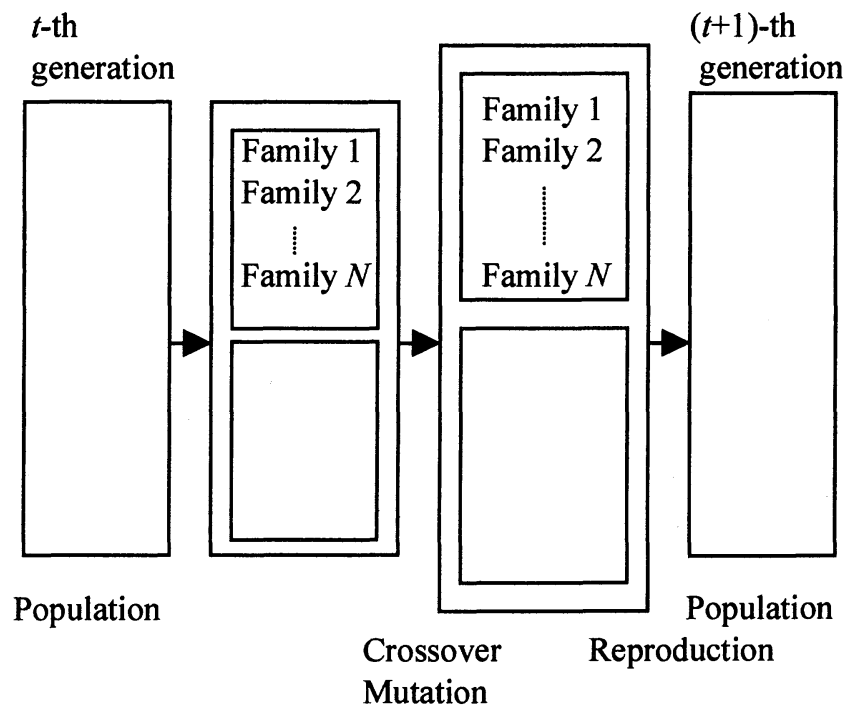

Figure 2 Introducing a family elitist concept in GA. 
Select individuals based on each objective function

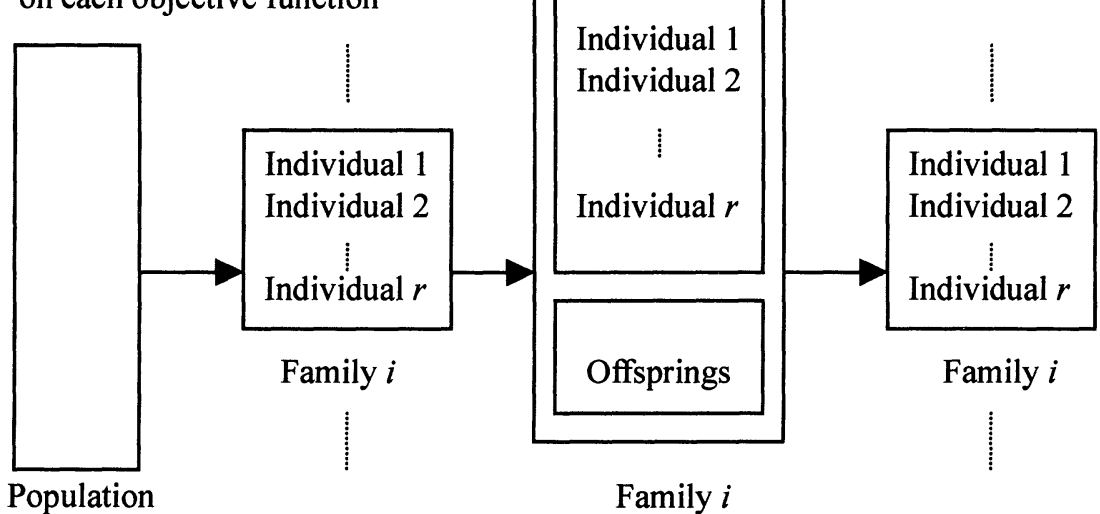

\begin{tabular}{l|l}
$\begin{array}{l}\text { Crossover } \\
\text { Mutation }\end{array}$ & Reproduction
\end{tabular}

Figure 3 Genetic operations in families.

\section{META-HEURISTIC SATISFICING TRADEOFF METHOD}

Satisficing tradeoff method is applicable for any problems if we could provide a method to solve min-max problems, but for a complex combinatorial multiobjective problems, it is hard to solve min-max problem. For a larger scale problem it is getting harder to solve min-max problem in a short time.

In this paper we propose a meta-heuristic satisficing tradeoff method. In this method we generate a set of Pareto optimal solutions (individuals) of a combinatorial multiobjective optimization problem by GA and use them as a candidate of solution of the min-max problem to be solved. That is, we choose a solution of minmax problem from a set of Pareto optimal solutions (individuals). Starting from this solution we try to pursue local search by a simulated annealing method. Tradeoff analysis among multiple objective function is performed by interaction with the decision maker. That is, a min-max solution is presented to the decision maker if he is satisfied with this solution. If he is not satisfied with this solution, we ask him to revise his aspiration level for each objective function. Then, we try to find another min-max solution from the Pareto optimal solutions (individuals). Figure 4 shows a flow of meta-heuristic satisficing tradeoff method.

In this meta-heuristic satisficing tradeoff method we just try to find a min-max solution from the Pareto optimal solutions (individuals) of the combinatorial multiobjective optimization problem instead of solving complex min-max problem in each iteration. Therefore, we could expect to get a satisfied preferred solution for the decision maker within a short computation time. Furthermore, by using family elitist strategy in GA for obtaining Pareto optimal solutions we could expect to get better compromise solution in a set of Pareto optimal solutions compared with the method without using family elitist strategy. 


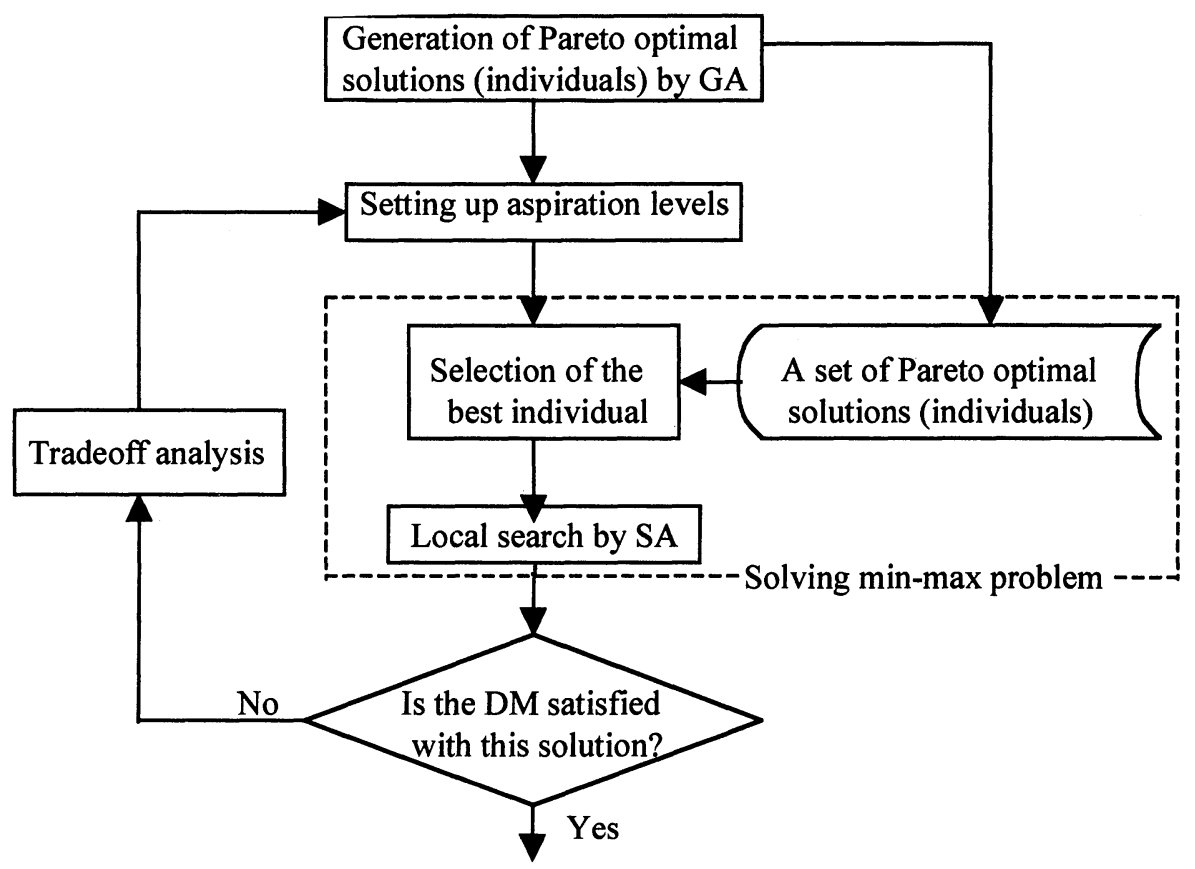

Figure 4 Flow of a meta-heuristic satisficing tradeoff method.

\section{FLOWSHOP SCHEDULING PROBLEM}

A flowshop scheduling problem in this paper has two cascade processes A and B. Process A has two parallel processing units 1 and 2. There exists no buffer between two processes. All the products are first processed at the Process A either by the processing unit 1 or 2 , and immediately right after this process they are processed at the Process B. There are four objectives to be minimized as follows:

(a) Minimize $f_{1}=$ total processing time

(b) Minimize $f_{2}=$ number of setups at Process A

(c) Minimize $f_{3}=$ the sum of variation rate of the products processed

(d) Minimize $f_{4}=$ the penalty for violating the continuous processing constraints

We solved a flowshop scheduling problems to process 100 products by the metaheuristic satisficing tradeoff method. We compared the method having family elitist strategy dealing with 25 families with the method having no family elitist strategy in the GA. We obtained better solution by the method having family elitist strategy.

\section{CONCLUDING REMARKS}

In this paper a meta-heuristic satisficing tradeoff method is proposed to solve multiobjective combinatorial optimization problems effectively. Since many performance evaluation problems in production management are multiobjective and 
combinatorial in nature, the method proposed in this paper is expected to be used effectively in many real production management systems. Further research problems to improve the method are how to set up aspiration levels, how to evaluate Pareto optimality of the individuals, application to many kinds of real problems, and so forth.

\section{REFERENCES}

Bedarahally, P.V., R.A. Perez and S. Chung (1996). A family elitist approach in genetic algorithms. Proc. 1996 ACM Symposium on Applied Computing, pp. 238-244, Philadelphia.

Cook, W.J., W.H. Cunningham, W.R. Pulleyblank and A. Schrijver (1998). Combinatorial Optimization, Wiley, New York.

Fonseca, C.M., and P.J. Fleming (1995). An overview of evolutionary algorithms in multiobjective optimization. Evolutionary Computation, Vol. 3, No. 1, pp. 1-16.

Goldberg, D.E. (1989). Genetic Algorithms in Search, Optimization \& Machine Learning. Addison Wesley, Reading, Mass.

Holland, J.H. (1975), Adaptation in Natural and Artificial Systems. University of Michigan Press, Ann Arbor.

Nakayama, H. and Y. Sawaragi (1984). Satisficing tradeoff method for multiobjective programming. in M. Grauer and A.P. Wierzbicki, eds.: Interactive Decision Analysis, Springer-Verlag, Berlin, pp. 113-122.

Tamaki, H., and E. Nishino (1998). A genetic algorithm approach to multiobjective scheduling problems with regular and non-regular objective functions. Proc. IFAC Symp. on Large Scale Systems: Theory and Applications (IFAC LSS'98), Rio, Patras, Greece, Vol. 1, pp. 289-294.

\section{BIOGRAPHY}

Dr. Hiroyuki Tamura is a professor at the Department of Systems and Human Science, Graduate School of Engineering Science, Osaka University, Japan. His research interests center on the systems planning methodology for large-scale complex systems and its applications to manufacturing systems and public systems.

Mr. Tomohiro Shibata received M.S. degree in engineering science from Osaka University in 1997. He is presently with Murata Corporation. While he was a graduate student at Osaka University, he was interested in modeling production scheduling problems and how to solve the problems.

Dr. Itsuo Hatono is an associate professor at the Information Processing Center, Kobe University, Japan. His research interests center on production scheduling and modeling and analysis of discrete event systems such as manufacturing systems and computer networks. 